\title{
PALFA Discovery of a Highly Relativistic Double Neutron Star Binary
}

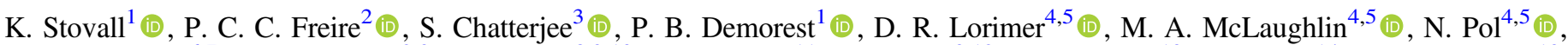

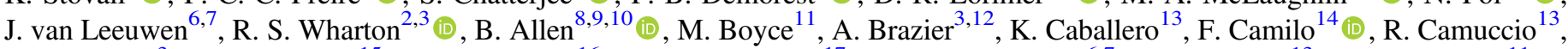

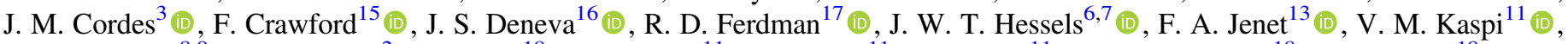

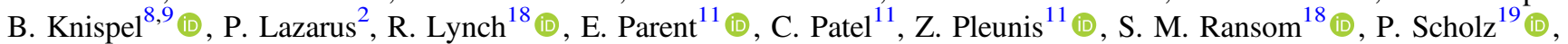
A. Seymour ${ }^{20}$, X. Siemens ${ }^{10}$, I. H. Stairs ${ }^{21}$ (i), J. Swiggum ${ }^{10}$ (i), and W. W. Zhu ${ }^{2,22}$ (i)

${ }^{1}$ National Radio Astronomy Observatory, 1003 Lopezville Road, Socorro, NM, 87801, USA; kstovall@nrao.edu 2 Max-Planck-Institut für Radioastronomie, Auf dem Hügel 69, D-53131 Bonn, Germany

${ }^{3}$ Cornell Center for Astrophysics and Planetary Science and Department of Astronomy, Cornell University, Ithaca, NY 14853, USA

${ }^{4}$ Dept. of Physics and Astronomy, West Virginia University, Morgantown, WV 26506, USA

${ }^{5}$ Center for Gravitational Waves and Cosmology, Chestnut Ridge Research Building, Morgantown, WV 26505, USA

${ }^{6}$ ASTRON, the Netherlands Institute for Radio Astronomy, Postbus 2, 7990 AA Dwingeloo, The Netherlands

${ }^{7}$ Anton Pannekoek Institute for Astronomy, University of Amsterdam, Science Park 904, 1098 XH Amsterdam, The Netherlands

${ }^{8}$ Max-Planck-Institut für Gravitationsphysik, D-30167 Hannover, Germany

${ }^{9}$ Leibniz Universität Hannover, D-30167 Hannover, Germany

${ }^{10}$ Physics Department, University of Wisconsin-Milwaukee, Milwaukee WI 53211, USA

${ }^{11}$ Department of Physics \& McGill Space Institute, McGill University, Montreal, QC H3A 2T8, Canada

${ }^{12}$ Cornell Center for Advanced Computing, Cornell University, Ithaca, NY 14850, USA

${ }^{13}$ Center for Advanced Radio Astronomy, University of Texas Rio Grande Valley, Brownsville, TX 78520, USA

${ }^{14}$ SKA South Africa, Pinelands 7405, South Africa

${ }^{15}$ Dept. of Physics and Astronomy, Franklin and Marshall College, Lancaster, PA 17604-3003, USA

${ }^{16}$ George Mason University, resident at the Naval Research Laboratory, 4555 Overlook Avenue SW, Washington, DC 20375, USA

${ }^{17}$ Faculty of Science, University of East Anglia, Norwich Research Park, Norwich NR4 7TJ, UK

${ }^{18}$ NRAO, Charlottesville, VA 22903, USA

${ }^{19}$ National Research Council of Canada, Herzberg Astronomy and Astrophysics, Dominion Radio Astrophysical Observatory, P.O. Box 248, Penticton, BC V2A 6J9, Canada

${ }^{20}$ Arecibo Observatory, HC3 Box 53995, Arecibo, PR 00612, USA

${ }^{21}$ Department of Physics and Astronomy, University of British Columbia, Vancouver, BC V6T 1Z1, Canada

22 National Astronomical Observatories, Chinese Academy of Sciences, A20 Datun Road, Chaoyang District, Beijing 100012, People's Republic of China Received 2018 January 5; revised 2018 January 31; accepted 2018 February 3; published 2018 February 16

\begin{abstract}
We report the discovery and initial follow-up of a double neutron star (DNS) system, PSR J1946+2052, with the Arecibo L-Band Feed Array pulsar (PALFA) survey. PSR J1946+2052 is a $17 \mathrm{~ms}$ pulsar in a $1.88 \mathrm{hr}$, eccentric ( $e=0.06$ ) orbit with a $\gtrsim 1.2 M_{\odot}$ companion. We have used the Jansky Very Large Array to localize PSR J1946 +2052 to a precision of 0 !" 09 using a new phase binning mode. We have searched multiwavelength catalogs for coincident sources but did not find any counterparts. The improved position enabled a measurement of the spin period derivative of the pulsar $\left(\dot{P}=9 \pm 2 \times 10^{-19}\right)$; the small inferred magnetic field strength at the surface $\left(B_{S}=4 \times 10^{9} \mathrm{G}\right)$ indicates that this pulsar has been recycled. This and the orbital eccentricity lead to the conclusion that PSR J1946+2052 is in a DNS system. Among all known radio pulsars in DNS systems, PSR J1946+2052 has the shortest orbital period and the shortest estimated merger timescale, 46 Myr; at that time it will display the largest spin effects on gravitational-wave waveforms of any such system discovered to date. We have measured the advance of periastron passage for this system, $\dot{\omega}=25.6 \pm 0.3 \mathrm{deg} \mathrm{yr}^{-1}$, implying a total system mass of only $2.50 \pm 0.04 M_{\odot}$, so it is among the lowest-mass DNS systems. This total mass measurement combined with the minimum companion mass constrains the pulsar mass to $\lesssim 1.3 M_{\odot}$.
\end{abstract}

Key words: pulsars: individual (PSR J1946+2052)

\section{Introduction}

Since the discovery of PSR B1913+16 (Hulse \& Taylor 1975), double neutron star (DNS) systems have allowed a wide range of investigations into many aspects of astrophysics and fundamental physics. Paramount among these have been tests of general relativity (GR) and alternative theories of gravity. The exquisite match between the observed rate of orbital decay of PSR B1913+16 and that predicted by GR due to the emission of gravitational waves (GWs; Damour \& Taylor 1991; Weisberg \& Huang 2016) showed that GR gives a self-consistent description of relativistic effects. Moreover, it established experimentally that GWs are not a mere coordinate effect: they carry energy across space and have a real effect on the orbital dynamics of massive objects. This indirect detection of GWs preceded the first direct detections (Abbott et al. 2016) by decades. The continued orbital decay in PSR B1913+16 inevitably leads to the neutron stars merging, and GWs from such a merger have recently been detected (Abbott et al. 2017).

Twelve more DNS systems have since been discovered, with another three DNS candidate systems unconfirmed (for a review, see Tauris et al. 2017). Several of these-PSRs J0737 -3039A/B (Burgay et al. 2003), B1534+12 (Fonseca et al. 2014), J1756-2251 (Ferdman et al. 2014), J1757 -1854 (Cameron et al. 2018), J1906+0746 (another PALFA discovery; Lorimer et al. 2006; van Leeuwen et al. 2015), and 
B2127+11C (Jacoby et al. 2006) - have also been used to test the predictions of theories of gravity.

Of these, J0737-3039A/B, the "double pulsar," has been the most outstanding test system. The discovery of the recycled pulsar in the system, PSR J0737-3039A, was in itself sufficient to significantly increase the estimated Galactic DNS merger rate (Burgay et al. 2003; Kalogera et al. 2004). The discovery that the second NS in the system is also a pulsar, PSR J0737-3039B (Lyne et al. 2004), allowed for a total of four independent and stringent tests of GR from timing observations alone (Kramer et al. 2006).

Given the extraordinary scientific results that have emerged from the study of DNS systems, their discovery has been an important motivation for many ongoing pulsar surveys. In this Letter, we focus on a discovery from the PALFA survey (Cordes et al. 2006; Lazarus et al. 2015), currently being carried out with the Arecibo Observatory. PALFA has thus far resulted in the discovery of 180 pulsars, including 22 millisecond pulsars (e.g., Deneva et al. 2012; Allen et al. 2013; Knispel et al. 2015; Scholz et al. 2015; Stovall et al. 2016), two DNS systems, PSRs J1906+0746 and J1913 +1102 (Lorimer et al. 2006; Lazarus et al. 2016), and a repeating fast radio burst (FRB; Spitler et al. 2016). In this Letter, we present the discovery of PSR J1946+2052, a $17 \mathrm{~ms}$ pulsar in a $1.88 \mathrm{hr}$, eccentric $(e=0.064)$ orbit with a $\sim 1.2 M_{\odot}$ companion. This is the third DNS system found in PALFA and is the DNS system with the shortest orbital period.

\section{Observations and Analysis}

The PALFA survey uses the Arecibo L-Band Feed Array (ALFA) receiver's 7 beams to search the Galactic plane $\left(b<\left|5^{\circ}\right|\right)$ visible by the Arecibo Observatory for pulsars and FRBs. The survey consists of two portions, on the inner $\left(32^{\circ}<l<77^{\circ}\right)$ and outer $\left(168^{\circ}<l<214^{\circ}\right)$ Galaxy. These are identical in setup except for the pointing integration time of $260 \mathrm{~s}$ for the inner versus $180 \mathrm{~s}$ for the outer Galaxy. The survey uses the Mock spectrometers centered at $1375.489 \mathrm{MHz}$ over $322.398 \mathrm{MHz}$ of bandwidth divided into 960 frequency channels, sampled every $65 \mu$ s (Lazarus et al. 2015).

\subsection{Discovery and Early Follow-up}

The PALFA survey identifies candidate discoveries using three separate pipelines: (1) a reduced time-resolution pipeline using the PRESTO software suite (Ransom 2001) without acceleration searching known as the "Quicklook" pipeline (Stovall 2013), (2) a full-resolution PRESTO pipeline with enhanced radio frequency interference (RFI) mitigation techniques and searches for acceleration up to $1650 \mathrm{~m} \mathrm{~s}^{-2}$ for a $10 \mathrm{~ms}$ pulsar (see Lazarus et al. 2015), and (3) an Einstein@ Home pipeline that searches for tight binaries using a templatematching search (Allen et al. 2013).

PSR J1946+2052 was discovered in pipeline (1), so we will briefly describe it here. The Quicklook pipeline is run on-site at the Arecibo Observatory to rapidly identify strong pulsar signals. Data from the Mock spectrometers are converted from 8-bit to 4-bit and the two sub-bands for each beam are combined into a single PSRFITS file as described in Section 3.2 of Lazarus et al. (2015). Each combined file is reduced in time-resolution by a factor of two and is examined by rfifind in order to create a RFI mask. The data are then de-dispersed at a series of trial DMs ranging from 0 to 1550.5 $\mathrm{pc} \mathrm{cm}^{-3}$ and searched for periodic signals using accelsearch summing up to 16 harmonics, but without searching for acceleration. The candidates are sorted by significance and the top 20 candidates are folded into diagnostic plots. The plots are examined using the PEACE algorithm (Lee et al. 2013) to identify the most promising candidates.

In PALFA observations recorded on 2017 July 19, inspection of the Quicklook pipeline diagnostic plots resulted in the discovery of a $17 \mathrm{~ms}$ pulsar with a significant apparent period change within the $260 \mathrm{~s}$ observation at a DM of about $94 \mathrm{pc} \mathrm{cm}^{-3}$. Subsequent Arecibo observations in 2017 August and September were performed using the L-wide receiver and the PUPPI backend configured in coherently de-dispersed search mode. The PUPPI observations were recorded at a center frequency of $1381 \mathrm{MHz}$ with a bandwidth of $800 \mathrm{MHz}$ across 512 frequency channels that were coherently dedispersed at the pulsar's best-known DM at the time of observation. Samples were recorded every $10.24 \mu$ s. We used some of these initial observations to determine the pulsar's orbit using PRESTO's fitorb.py and created a preliminary ephemeris. Observations were folded using this ephemeris into $10 \mathrm{~s}$ subintegrations, cleaned of RFI, and reduced to two frequency channels and $60 \mathrm{~s}$ subintegrations. The pulse profile for PSR J1946+2052 from a $2 \mathrm{hr}$ observation is shown in Figure 1. This observation has been polarization- and fluxcalibrated by scaling the pulsar observation using an observation of a noise diode injected signal and an unpolarized quasar $(\mathrm{J} 1445+0958)$. No polarization has been detected in PSR $\mathrm{J} 1946+2052$, so we only show total intensity. We generated a template for the characteristic pulse shape of PSR J1946+2052 by summing 40 minutes of observations together and smoothing the summed profile. Times-of-arrival (TOAs) were then generated by cross-correlating each frequency channel for every 60 s subintegration in the Fourier domain (Taylor 1992) with our template using the PSRCHIVE tool pat (Hotan et al. 2004). The resulting TOAs were compared to a model for PSR J1946+2052 using the tempo pulsar timing software.

On 2017 September 28, we used the Robert C. Byrd Green Bank Telescope to observe PSR J1946+2052 at $820 \mathrm{MHz}$ using GUPPI (DuPlain et al. 2008) with a bandwidth of $200 \mathrm{MHz}$ across 128 frequency channels in coherent search mode with a sample time of $10.24 \mu \mathrm{s}$. We used the same procedure as above to obtain TOAs. To properly account for the time offset between the GBT-GUPPI observations and Arecibo-PUPPI observations, we used offsets measured by the NANOGrav collaboration from observations of PSR J1713+0747 (Arzoumanian et al. 2015).

Since we have been recording follow-up data in coherent search mode with GUPPI and PUPPI, this has enabled for us to search for a potential companion pulsar. Thus far we have not detected the companion as a pulsar.

\subsection{Localization}

ALFA has a beam size (FWHM) of 3!35 (Cordes et al. 2006); this represents the approximate uncertainty in the sky location of the pulsar at the time of discovery. To better localize the pulsar, we observed its approximate position using the Karl G. Jansky Very Large Array (VLA) during the move from $\mathrm{C}$ to $\mathrm{B}$ configuration; however, most of the antennas were already in their locations for B configuration. We observed at L-band from 1 to $2 \mathrm{GHz}$ on 2017 September 3 and S-band from 2 to $4 \mathrm{GHz}$ on 2017 September 6. For both observations, data were recorded in a new imaging mode in which the correlation (visibility) data are 


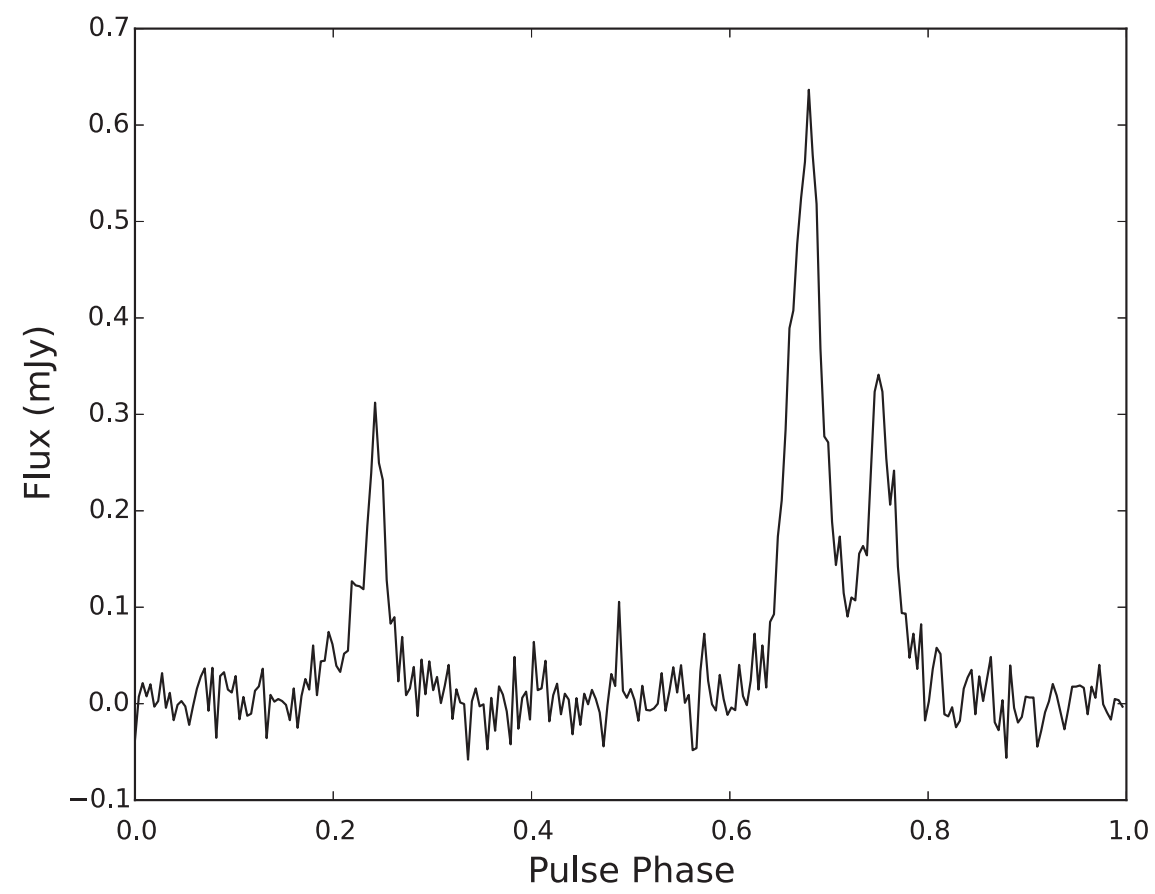

Figure 1. Average profile for PSR J1946+2052 at $1.43 \mathrm{GHz}$ from a $2 \mathrm{hr}$ observation from the Arecibo Observatory using the PUPPI backend in coherent search mode.
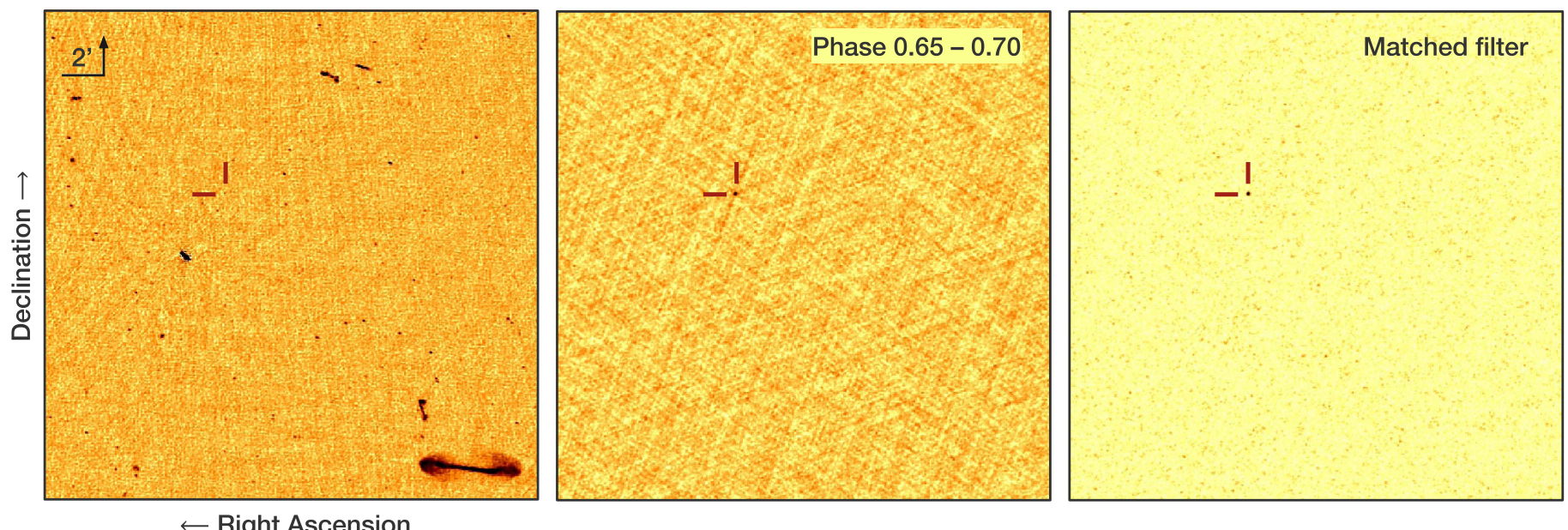

$\leftarrow$ Right Ascension

Figure 2. Left: bin-averaged image from 1 to $2 \mathrm{GHz}$ of the field with PSR J1946+2052. Center: zoomed-in version of left plot showing only one of the phase bins; here, the pulsar becomes obvious. Right: image showing the maximum of the convolution with the template profile for each pixel.

integrated separately into 20 pulse phase ranges ("bins") based on the pulsar's initial timing ephemeris. We de-dispersed and subtracted the mean, period-averaged signal in the visibility domain using sdmpy, ${ }^{23}$ then calibrated and imaged each bin individually using CASA. ${ }^{24}$ The mean-subtraction removes all continuous sources from the image, while the pulsar's signal remains as it is peaked at a small subset of phase bins. We then convolved the set of images versus bin with the pulsar's profile template and recorded the maximum value of the convolution for each image pixel, effectively performing a matched filter. The pulsar was not detectable at S-band, but the L-band matched filtered data resulted in a clear detection (see Figure 2, right). From the matched-filter image, we find the position of PSR J1946+2052 to be 19:46:14.130(6) +20:52:24.64(9). We fit the

\footnotetext{
23 http://github.com/demorest/sdmpy

24 http://casa.nrao.edu
}

DD binary model (Damour \& Deruelle 1985, 1986) with the pulsar position fixed to that measured from the VLA localization using tempo. The resulting timing solution is shown in Table 1.

\subsection{Multiwavelength Analysis}

Once the localization was achieved, we examined multiple data archives at the measured interferometric position and found a nearby source in IR (UKIDSS) and optical (IPHAS and SDSS) images. The nearby source is named J194614.14 +205224.7 in the UKIDSS Galactic Plane Survey (UGPS; Lucas et al. 2008) and is 0 "' 175 away from the VLA position of $\mathrm{J} 1946+2052$. The IPHAS (Drew et al. 2005) source is identified as J194614.14+205224.5 and is 0". 16 away from the position of J1946+2052. We calculated the probability of such a chance coincidence using the IPHAS source counts to be $\sim 0.002$. However, in both catalogs the source is identified as a 
Table 1

Fitted and Derived Parameters for PSR J1946+2052

\begin{tabular}{|c|c|}
\hline \multicolumn{2}{|l|}{ Measured Parameters } \\
\hline R.A., $\alpha$ (J2000.0) & $19: 46: 14.130(6)^{\mathrm{a}}$ \\
\hline Decl., $\delta(\mathrm{J} 2000.0)$ & $20: 52: 24.64(9)^{\mathrm{a}}$ \\
\hline Pulse frequency, $\nu\left(\mathrm{s}^{-1}\right)$ & $58.9616546384(5)$ \\
\hline First derivative of pulse frequency, $\dot{\nu}\left(\mathrm{s}^{-2}\right)$ & $-3.2(6) \times 10^{-15}$ \\
\hline Epoch (MJD) & 57989.0 \\
\hline Dispersion measure, DM $\left(\mathrm{pc} \mathrm{cm}^{-3}\right)$ & $93.965(3)$ \\
\hline Ephemeris & DE436 \\
\hline Clock & TT(BIPM) \\
\hline Span of Timing Data (MJD) & $57953-58024$ \\
\hline rms Residual $(\mu s)$ & 95.04 \\
\hline Binary model & DD \\
\hline Orbital period, $P_{\mathrm{b}}$ (days) & $0.07848804(1)$ \\
\hline Projected semimajor axis, $x$ (lt-s) & $1.154319(5)$ \\
\hline Orbital eccentricity, $e$ & $0.063848(9)$ \\
\hline Epoch of periastron, $T_{0}(\mathrm{MJD})$ & $57989.002943(3)$ \\
\hline Longitude of periastron, $\omega$ (degrees) & $132.88(1)$ \\
\hline Rate of periastron advance, $\dot{\omega}\left(\right.$ degrees $\left.\mathrm{yr}^{-1}\right)$ & 25.6(3) \\
\hline $1400 \mathrm{MHz}$ mean flux density Arecibo ( $\mu \mathrm{Jy})$ & $62(6)$ \\
\hline $1400 \mathrm{MHz}$ mean flux density VLA ( $\mu \mathrm{Jy})$ & $84(15)$ \\
\hline \multicolumn{2}{|l|}{ Derived Parameters } \\
\hline Galactic latitude, $l$ (degrees) & 57.66 \\
\hline Galactic longitude, $b$ (degrees) & -1.98 \\
\hline DM-derived distance (NE2001), $d_{\mathrm{DM}}(\mathrm{kpc})$ & 4.2 \\
\hline DM-derived distance (YMW16), $d_{\mathrm{DM}}(\mathrm{kpc})$ & 3.5 \\
\hline Spin period, $P(\mathrm{~s})$ & $0.0169601753230(2)$ \\
\hline Spin period derivative, $\dot{P}$ & $9(2) \times 10^{-19}$ \\
\hline Characteristic age, $\tau_{\mathrm{c}}=P / 2 \dot{P}(\mathrm{Myr})$ & 290 \\
\hline Surface magnetic field, $B_{\mathrm{S}}=3 \times 10^{19} \sqrt{P \dot{P}}\left(10^{9} \mathrm{G}\right)$ & 4 \\
\hline Spin-down luminosity $\left(10^{32} \mathrm{erg} \mathrm{s}^{-1}\right)$ & 75 \\
\hline Mass function, $f_{\text {mass }}\left(M_{\odot}\right)$ & $0.268184(12)$ \\
\hline Total mass, $M_{\text {Total }}\left(M_{\odot}\right)$ & $2.50(4)$ \\
\hline
\end{tabular}

Note. Numbers in parentheses represent $1 \sigma$ uncertainties from tempo, scaled for reduced $\chi^{2}=1$.

${ }^{a}$ VLA positions, fixed in the tempo fit.

galaxy based on the source being extended. We also checked and did not see any indication of a source at the location of PSR J1946+2052 in 2MASS, DSS, GALEX, ROSAT, and Fermi archival data.

\section{Discussion}

\subsection{Formation and Nature of the System}

Through the precise localization of PSR J1946+2052, the period derivative could be determined to be $9(2) \times 10^{-19} \mathrm{~s} \mathrm{~s}^{-1}$ after only 71 days of timing. Ignoring the effects from the Shklovskii effect and Galactic acceleration that are expected to be very small for PSR J1946+2052, this suggests a characteristic age of $290 \mathrm{Myr}$ and surface dipole magnetic field of $4 \times 10^{9} \mathrm{G}$. The small spin-down rate indicates the pulsar was recycled. The implied matter transfer from the companion progenitor would also have circularized the orbit. If the companion had evolved to a white dwarf, the system would have retained a nearly circular orbit, as observed for nearly all pulsar-white dwarf systems. We measure, however, a significant system eccentricity $e=0.064$, which requires a kick and/or sudden mass loss associated with the supernova $(\mathrm{SN})$ of the progenitor of the companion. This evidence, coupled with the mass fraction, indicates that the companion is an NS and the system a DNS.
In almost all aspects of its measured orbital parameters, the PSR J1946+2052 DNS resembles a further evolved version of the double pulsar J0737-3039A/B (Burgay et al. 2003). Below, we discuss the implications of this similarity, assuming the companion mass is the same as in the case of the double pulsar $\left(m_{2} \sim 1.25 M_{\odot}\right)$. In that case, the constraint on the total mass of the system (see Section 3.2) yields $m_{1}=1.25 M_{\odot}$.

Integrating the equations for the orbital decay back $290 \mathrm{Myr}$ we derive firm upper limits on the orbital eccentricity and period at birth of $e<0.14$ and $P_{b}<0.17$ days. As in the double pulsar (Lorimer et al. 2007), these indicate that these systems had a small separation before the second SN.

Low eccentricities at birth imply small kicks associated with the second SN. In the case of the double pulsar, the eccentricity, proper motion, and misalignment between the spin and orbital axes $\left(<3^{\circ}\right.$; see Ferdman et al. 2013) are all very small. That conclusively limits the second SN kick to $\sim 70 \mathrm{~km} \mathrm{~s}^{-1}$ (e.g., Piran \& Shaviv 2005). This small kick suggests a close binary interaction prior to the second $\mathrm{SN}$, which stripped off the envelope of the evolving secondary. Such ultra-stripped SNe often have smaller kicks, which seem to be associated with the formation of lighter NSs (Tauris et al. 2017).

If the companion of PSR J1946+2052 has also originated in such a low-kick SN, then we should expect the system to be similar to the double pulsar: a small value for $m_{2}$, a small peculiar velocity (the system would have a low velocity relative to the local standard of rest, LSR) and a relatively close alignment between the spin axis and the orbital angular momentum for PSR J1946+2052. As in PSR J0737-3039A (Ferdman et al. 2013), we should not expect any pulse-profile changes due to geodetic precession. We are testing these predictions with continued observations.

\subsection{Measurement and Prediction of Post-Keplerian Parameters and System Masses}

Of all pulsars known in DNSs, PSR J1946+2052 has the shortest orbital period. It also has the largest rate of advance of periastron, $\dot{\omega}=25^{\circ} .6 \mathrm{yr}^{-1}$. Still, if this advance solely is due to GR (Robertson 1938; Taylor \& Weisberg 1982), the inferred total mass of $M_{\text {Total }}=2.50 \pm 0.04 M_{\odot}$ is potentially less than the lightest DNS known, PSR J1411+2551 (Martinez et al. 2017). From $M_{\text {Total }}$ and the mass function $\left(f=0.268184(12) M_{\odot}\right)$ we derive an upper limit for the mass of the pulsar $\left(m_{1}<1.31 M_{\odot}\right)$ and lower limit for the companion mass $\left(m_{2}>1.18 M_{\odot}\right)$; see Figure 3.

Without further PK parameters we cannot yet determine the individual NS masses. For $m_{1}=1.25 M_{\odot}$ and $m_{2}=1.25 M_{\odot}$ we expect an Einstein delay $\gamma=0.262 \mathrm{~ms}$. That is small compared to other DNS systems, but nevertheless simulations indicate that continued timing will measure $\gamma$ with $\sim 10 \%$ and $\sim 1 \%$ relative uncertainty by mid-2019 and mid-2025, respectively. This will allow a precise measurement of both masses. Furthermore, for the masses assumed above, GR predicts an orbital decay due to the emission of GWs of $\dot{P}_{b}=-1.78 \times 10^{-12} \mathrm{~s} \mathrm{~s}^{-1}$. This will be measured with a relative uncertainty of $\sim 7.5 \%$ and $\sim 0.2 \%$ by mid-2019 and mid-2025, respectively.

\subsection{PSR J1946+2052 as a Gravitational Laboratory}

The measurement of $\dot{P}_{b}$ will be contaminated by two kinematic effects: first, by the difference in Galactic acceleration of the solar system and the pulsar (Nice \& Taylor 1995; estimated 


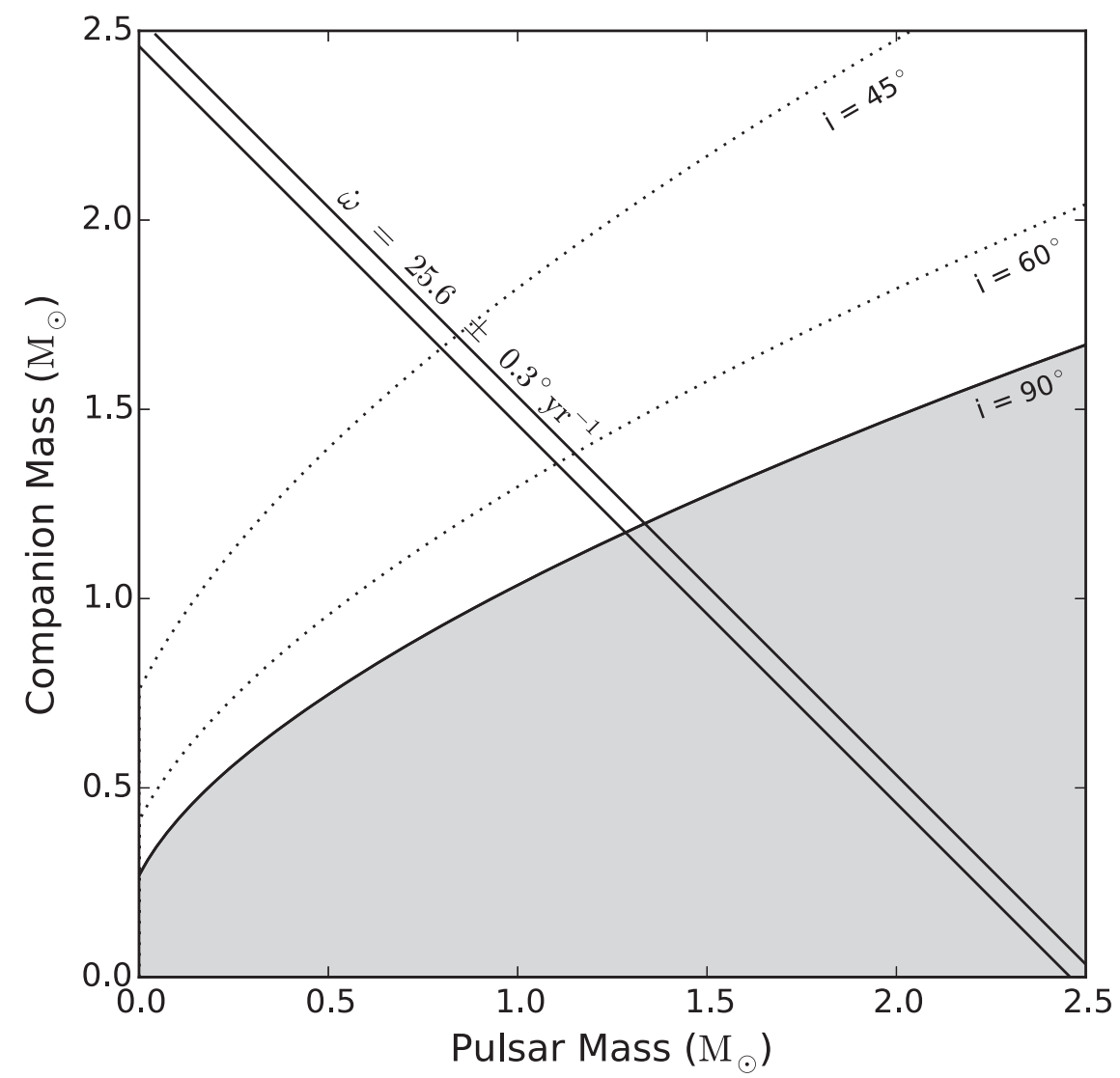

Figure 3. Possible values for the mass of the pulsar and companion. The gray region is not allowed due to the mass function. The solid lines show the constraints due to the measurement of $\dot{\omega}$, as given by GR. The dotted lines represent varying orbital inclination angles $(i)$.

below using the Galactic parameters from Reid et al. 2014) and second by the pulsar proper motion (Shklovskii 1970). These can be corrected once the distance is known. The NE2001 (Cordes \& Lazio 2002) and YMW16 (Yao et al. 2017) models predict distances of 4.2 and $3.5 \mathrm{kpc}$, respectively. Given that and the faintness of the pulsar, a precise distance from VLBI or HI absorption (as done for PSR J1906+0746 in van Leeuwen et al. 2015) appears unlikely in the near future. Nevertheless, it is possible to estimate these kinematic contributions, assuming a

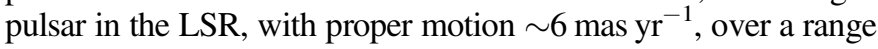
of distances of $4.2 \pm 1 \mathrm{kpc}$. The sum of the kinematic contributions to $\dot{P}_{b}$ for $d=3.2$ and $5.2 \mathrm{kpc}$ changes by only $+2.1 /-2.6 \times 10^{-16} \mathrm{~s} \mathrm{~s}^{-1}$. With such a small uncertainty, the GR prediction for the orbital decay can be tested to a precision of $0.015 \%$. This is one order of magnitude better than the $0.16 \%$ test possible with the Hulse-Taylor pulsar (Weisberg \& Huang 2016). The quality of the PSR J1946+2052 test will depend very significantly on its proper motion, making its measurement an important objective of future timing.

\subsection{Implications of PSR J1946+2052 on the DNS Merger Rate}

The large expected rate of orbital decay implies the system will merge quickly. Indeed, based on the measured orbit and total mass, and the likely component masses, the coalescence timescale is only $46 \mathrm{Myr}$. That is significantly shorter than the coalescence timescales of the double pulsar (85 Myr) and PSR J1757-1854 (76 Myr). The current mean GW luminosity of PSR J1946+2052 is the largest of any known DNS: $~ 13 \%$ of a solar luminosity (compared to $6.2 \%$ for the double pulsar and $10.8 \%$ for PSR J1757-1854).
Since PSR J1946+2052 strongly resembles a more evolved double pulsar system, we assume they belong to similar populations of DNS binaries. We thus use existing population models (Kim et al. 2015) to calculate how many J0737-3039like binaries the PALFA survey can detect. We use PsrPopPy ${ }^{25}$ (Bates et al. 2014) to perform the population synthesis and analysis.

The total number of these systems in the Galaxy is $N_{\text {pop }}=1500_{-1000}^{+4000}(95 \%$ confidence interval; Kim et al. 2015). Using PsrPopPy, we generated a population of $N_{\text {pop }}$ pulsars with the same spin period and orbital parameters as PSR J1946+2052. We found that given this population, the PALFA survey should have detected $2_{-1}^{+5}$ DNS systems like J1946+2052, to date. Therefore, the discovery of PSR J1946+2052 is predicted by the population models used for current merger rate estimates and is unlikely to dramatically change the most recently published rate of $\mathcal{R}_{\mathrm{g}}=21_{-14}^{+28} \mathrm{Myr}^{-1}$ (Kim et al. 2015).

\subsection{Spin Effects during the Merger}

The small spin period, its derivative, and the relatively short coalescence time imply that the pulsar will still be spinning rapidly when it merges. For braking indices between 0 and 3, the values vary between 17.9 and $18.5 \mathrm{~ms}$. For the larger spin period, the pulsar's dimensionless spin parameter at merger

\footnotetext{
25 https://github.com/samb8s/PsrPopPy
} 
will be given by

$$
\chi=\frac{c}{G} \frac{2 \pi I}{m_{1}^{2} P}=0.032,
$$

where we have assumed $m_{1}=1.25 M_{\odot}$ and a moment of inertia $I=1.25 \times 10^{45} \mathrm{~g} \mathrm{~cm}^{2}$ (Zhu et al. 2017). This is the largest $\chi$ at merger for any pulsar in a confirmed DNS system including PSR J1757-1854 (Zhu et al. 2017). This large spin parameter has implications for the ability to determine neutron star masses from the GW signals during NS-NS inspirals. As shown in Table 1 and Figure 4 of Abbott et al. (2017), a constraint on $\chi$ is necessary to precisely determine the masses of the individual NSs in a DNS merger. With $|\chi| \leqslant 0.05$ the primary and secondary NS masses are $1.36-1.60 M_{\odot}$ and $1.17-1.36 M_{\odot}$, respectively; with $|\chi| \leqslant 0.89$ constraint the limits are 1.36-2.26 $M_{\odot}$ and $0.86-1.36 M_{\odot}$, respectively. Thus, knowing the plausible range of values for $\chi$ at merger is important for estimating masses from GW observations of DNS mergers.

The Arecibo Observatory is operated by SRI International under a cooperative agreement with the National Science Foundation (NSF; AST-1100968), and in alliance with Ana G. Méndez-Universidad Metropolitana, and the Universities Space Research Association. The National Radio Astronomy Observatory is a facility of the NSF operated under cooperative agreement by Associated Universities, Inc. K.S., A.B., S.C., J.M.C., P.D., M.A.M., S.M.R., and F.C. are (partially) supported by the NANOGrav Physics Frontiers Center (NSF award 1430284). N.P. and M.A.M. are supported by NSF award number 1517003. I.H.S. and V.M.K. both acknowledge NSERC Discovery Grants and the Canadian Institute for Advanced Research (CIFAR). V.M.K. received further support from an NSERC Discovery Accelerator Supplement, NSERC's Harzberg Award, the Canada Research Chairs Program, and the Lorne Trottier Chair in Astrophysics and Cosmology. S.M.R. is a CIFAR Senior Fellow. P.C.C.F., J.v.L., R.S.W., and J.W.T.H. gratefully acknowledge financial support by the European Research Council, under the European Union's Seventh Framework Programme (FP/2007-2013) grant agreements 279702 (BEACON), 617199 (ALERT), 610058 (BLACKHOLECAM), and 337062 (DRAGNET), respectively. P.C.C.F. further acknowledges support from the Max Planck Society; J.W.T.H. from a NWO Vidi fellowship; J.S.D. was supported by the NASA Fermi program; and W.W.Z. by the Chinese Academy of Science Pioneer Hundred Talents Program and the National Key R\&D Program of China No. 2017YFA0402600. We thank Lijing Shao and Nobert Wex for stimulating discussions and useful suggestions.

Facilities: Arecibo, EVLA, GBT.

Software: PRESTO, PSRCHIVE, TEMPO, PsrPopPy, sdmpy, CASA.

\section{ORCID iDs}

K. Stovall (iD https://orcid.org/0000-0002-7261-594X

P. C. C. Freire (iD https://orcid.org/0000-0003-1307-9435

S. Chatterjee (iD https://orcid.org/0000-0002-2878-1502

P. B. Demorest (D) https://orcid.org/0000-0002-6664-965X

D. R. Lorimer (iD https://orcid.org/0000-0003-1301-966X

M. A. McLaughlin (D) https://orcid.org/0000-0001-7697-7422

N. Pol (i) https://orcid.org/0000-0002-8826-1285

R. S. Wharton (i) https://orcid.org/0000-0002-7416-5209
B. Allen (iD https://orcid.org/0000-0003-4285-6256

F. Camilo (i) https://orcid.org/0000-0002-1873-3718

J. M. Cordes (1) https://orcid.org/0000-0002-4049-1882

F. Crawford (i) https://orcid.org/0000-0002-2578-0360

J. S. Deneva (1D https://orcid.org/0000-0003-1226-0793

R. D. Ferdman (i) https://orcid.org/0000-0002-2223-1235

J. W. T. Hessels (i) https://orcid.org/0000-0003-2317-1446

F. A. Jenet (iD https://orcid.org/0000-0003-4990-1523

V. M. Kaspi (iD https://orcid.org/0000-0001-9345-0307

B. Knispel (i) https://orcid.org/0000-0003-3168-0929

R. Lynch (iD https://orcid.org/0000-0001-5229-7430

E. Parent (iD https://orcid.org/0000-0002-0430-6504

Z. Pleunis (iD https://orcid.org/0000-0002-4795-697X

S. M. Ransom (i) https://orcid.org/0000-0001-5799-9714

P. Scholz (i) https://orcid.org/0000-0002-7374-7119

I. H. Stairs (D) https://orcid.org/0000-0001-9784-8670

J. Swiggum (ib https://orcid.org/0000-0002-1075-3837

W. W. Zhu (iD https://orcid.org/0000-0001-5105-4058

\section{References}

Abbott, B. P., Abbott, R., Abbott, T. D., et al. 2016, PhRvL, 116, 061102 Abbott, B. P., Abbott, R., Abbott, T. D., et al. 2017, PhRvL, 119, 161101 Allen, B., Knispel, B., Cordes, J. M., et al. 2013, ApJ, 773, 91 Arzoumanian, Z., Brazier, A., Burke-Spolaor, S., et al. 2015, ApJ, 813, 65 Bates, S. D., Lorimer, D. R., Rane, A., \& Swiggum, J. 2014, MNRAS, 439, 2893 Burgay, M., D’Amico, N., Possenti, A., et al. 2003, Natur, 426, 531 Cameron, A. D., Champion, D. J., Kramer, M., et al. 2018, MNRAS, 475, L57 Cordes, J. M., Freire, P. C. C., Lorimer, D. R., et al. 2006, ApJ, 637, 446 Cordes, J. M., \& Lazio, T. J. W. 2002, arXiv:astro-ph/0207156

Damour, T., \& Deruelle, N. 1985, AIHS, 43, 107

Damour, T., \& Deruelle, N. 1986, AIHS, 44, 263

Damour, T., \& Taylor, J. H. 1991, ApJ, 366, 501

Deneva, J. S., Freire, P. C. C., Cordes, J. M., et al. 2012, ApJ, 757, 89

Drew, J. E., Greimel, R., Irwin, M. J., et al. 2005, MNRAS, 362, 753

DuPlain, R., Ransom, S., Demorest, P., et al. 2008, Proc. SPIE, 7019, 70191D

Ferdman, R. D., Stairs, I. H., Kramer, M., et al. 2013, ApJ, 767, 85

Ferdman, R. D., Stairs, I. H., Kramer, M., et al. 2014, MNRAS, 443, 2183 Fonseca, E., Stairs, I. H., \& Thorsett, S. E. 2014, ApJ, 787, 82

Hotan, A. W., van Straten, W., \& Manchester, R. N. 2004, PASA, 21, 302 Hulse, R. A., \& Taylor, J. H. 1975, ApJL, 195, L51

Jacoby, B. A., Cameron, P. B., Jenet, F. A., et al. 2006, ApJL, 644, L113 Kalogera, V., Kim, C., Lorimer, D. R., et al. 2004, ApJL, 601, L179

Kim, C., Perera, B. B. P., \& McLaughlin, M. A. 2015, MNRAS, 448, 928

Knispel, B., Lyne, A. G., Stappers, B. W., et al. 2015, ApJ, 806, 140

Kramer, M., Stairs, I. H., Manchester, R. N., et al. 2006, Sci, 314, 97 Lazarus, P., Brazier, A., Hessels, J. W. T., et al. 2015, ApJ, 812, 81 Lazarus, P., Freire, P. C. C., Allen, B., et al. 2016, ApJ, 831, 150 Lee, K. J., Stovall, K., Jenet, F. A., et al. 2013, MNRAS, 433, 688 Lorimer, D. R., Freire, P. C. C., Stairs, I. H., et al. 2007, MNRAS, 379, 1217 Lorimer, D. R., Stairs, I. H., Freire, P. C., et al. 2006, ApJ, 640, 428 Lucas, P. W., Hoare, M. G., Longmore, A., et al. 2008, MNRAS, 391, 136 Lyne, A. G., Burgay, M., Kramer, M., et al. 2004, Sci, 303, 1153 Martinez, J. G., Stovall, K., Freire, P. C. C., et al. 2017, ApJL, 851, L29 Nice, D. J., \& Taylor, J. H. 1995, ApJ, 441, 429

Piran, T., \& Shaviv, N. J. 2005, PhRvL, 94, 051102 Ransom, S. M. 2001, PhD thesis, Harvard Univ.

Reid, M. J., Menten, K. M., Brunthaler, A., et al. 2014, ApJ, 783, 130 Robertson, H. P. 1938, AnMat, 38, 101

Scholz, P., Kaspi, V. M., Lyne, A. G., et al. 2015, ApJ, 800, 123 Shklovskii, I. S. 1970, SvA, 13, 562

Spitler, L. G., Scholz, P., Hessels, J. W. T., et al. 2016, Natur, 531, 202 Stovall, K. 2013, PhD thesis, Univ. Texas

Stovall, K., Allen, B., Bogdanov, S., et al. 2016, ApJ, 833, 192

Tauris, T. M., Kramer, M., Freire, P. C. C., et al. 2017, ApJ, 846, 170 Taylor, J. H. 1992, RSPTA, 341, 117

Taylor, J. H., \& Weisberg, J. M. 1982, ApJ, 253, 908 van Leeuwen, J., Kasian, L., Stairs, I. H., et al. 2015, ApJ, 798, 118 Weisberg, J. M., \& Huang, Y. 2016, ApJ, 829, 55

Yao, J. M., Manchester, R. N., \& Wang, N. 2017, ApJ, 835, 29

Zhu, X.-J., Thrane, E., Osłowski, S., Levin, Y., \& Lasky, P. D. 2017, arXiv:1711.09226 